\title{
Molecular modeling studies for exploring structural requirement of acetylcholinesterase inhibitors
}

\author{
Tabassum Hossain, Achintya Saha*
}

\begin{abstract}
Inhibition of the neurotransmitter acetylcholine (ACh) can control the alzheimer's disease (AD). The ACh hydrolyzes to produce choline and acetyl groups through acetylcholinesterase (AChE) and butyrylcholinesterase (BChE) in the synaptic region, which play a key role in accelerating senile amyloid $\beta$-peptide (Aß) plaque depositions, leads to generation of $\mathrm{AD}$. The present study has been emphasized to explore both ligand- and structure-based QSAR and docking studies on a set of structurally diverse compounds to explore prime structural features responsible for selective binding to AChE, vis-a-vis inhibiting enzyme activity. Both the studies showed the importance of $\mathrm{HB}$ acceptor and donor, and hydrophobic features of the molecule for effective binding. Systematic comparisons revealed that structure-based study has advantages in efficiently identifying potent hits with structural diversity over simple ligand-based study. Structurebased QSAR study (site score $=1.006$ ) adjudged the significance of features obtained from ligand-based QSAR model $($ ROC score $=\mathbf{0 . 8 5 0}$, sensitivity $=\mathbf{0 . 7 1 0}$, specificity $=$ 0.932). Presence of electronegative groups, and acyclic and aromatic rings in the molecular scaffold depict the importance in selective AChE inhibition.
\end{abstract}

Keywords - AChE, Bayesian model, docking, Site Map analysis

\section{Introduction}

Deficiency in cholinergic neurotransmission is significant for the progression of Alzheimer's disease (AD). The ACh hydrolyzes to produce choline and acetyl group through the biocatalyst acetylcholinesterase (AChE) and butyrylcholinesterase (BChE) in the synaptic region [1]. Inhibition of AChE enzyme can sustain the ACh depletion, which is the main strategy for the treatment of $\mathrm{AD}$. In the present study, a group of compounds are considered for the molecular modeling studies to explore the structural requirements through ligand- and structure-based quantitative structure activity relationship (QSAR), site map, molecular docking studies for potential AChE inhibition.

\section{Tabassum Hossain}

Department of Chemical Technology

University of Calcutta

India

\section{Achintya Saha}

Department of Chemical Technology

University of Calcutta

India

\section{Materials and Methods}

In this work, a set of structurally diverse compounds ( $\mathrm{n}=$ 225) [2-17], having AChE inhibitory activity ( $\mathrm{IC}_{50}$ in $\mathrm{nM}$ ), have been considered for modeling study. The compounds were divided into training and test sets by k-means clustering. The crystal structure of the AChE enzyme (PDB code: 4EY7) [18], obtained from RCSB Protein Data Bank [19], has been used for structure-based modeling, site map and docking studies. The QSAR models were generated for understanding of steric (s), electrostatic (e), hydrophobic (p), ring aromatic (r), HB acceptor (a) and donor (d) requirement for enzyme inhibition.

\section{A. Bayesian model}

Development of Bayesian model [20, 21] helps to classify active and inactive compounds. The model was developed by the help of "Create Bayesian model" tool in Discover Studio 2.5 (DS 2.5) [22]. The 2D descriptors selected for model development were molecular function class fingerprints of maximum diameter 6 (FCFP_6), AlogP, molecular weight, number of rotatable bonds, number of rings, number of aromatic rings, number of hydrogen bond donors and acceptors, and molecular fractional polar surface area. The developed model emphasized some good fingerprints which are favourable for AchE inhibition and some bad fingerprints, the presence of which in the molecules may reduce the enzyme activity. The external predictability of the model was tested on the test set compounds.

\section{B. Molecular docking}

Molecular docking was carried out to find out the binding interaction of the small molecule at the active site of protein by using Grid-Based Ligand Docking with Glide in Schrodinger [23, 24]. Protein was prepared for experimentation using "Protein preparation wizard" module [25]. Hydrogen atoms were added, followed by energy minimization and optimization by OPLS force field. Protonation states were determined at physiological $\mathrm{pH} 7.4$ using PROPKA. Water molecules around the catalytic zone upto $5 \AA$ were kept during protein preparation and rest of the water molecules were removed. The ligand structures were prepared by the ligand preparation LigPrep module. The grid was generated according to the active site of the protein, after ligand and protein preparation. The gridenclosing box was centered to the active sites of the corresponding 3D-structure of the receptor so as to enclose them within $3 \AA$ from the centroid of these residues. Finally, docking calculation was performed with XP mode. Glide XP mode determines all reasonable orientation for each lowenergy conformer in the designated binding site. 


\section{Site map analyses}

The crystal structure of protein 4EY7 [18] was selected, and the site recognition tool 'Site Map' [26] of Maestro was run on the protein structure after extracting the ligand. Site Map applies an algorithm similar to Goodford's Grid algorithm [27] that uses interaction energies between protein residues and grid probes to generate preferable interaction sites. Sites containing more than 15 points were considered as druggable pockets. A restrictive hydrophobicity definition, a standard grid $(1.0 \AA)$, and OPLS force field were used. The physiological properties were calculated by Site Maps that, include size, volume, degree of contact, hydrophobic/hydrophilic balance, and hydrogen bonding possibilities (acceptors/donors). In addition, two scores SiteScore and Dscore were calculated. Site-Score determines the druggability of the selected pocket. A pocket having SiteScore of 0.8 may be considered as druggable, whereas a value of more than 1.0 is indicative of the highly promising site. The Dscore parameter penalizes highly hydrophobic sites since these sites are not suitable for polar ligands. The Site Map analysis has been carried out to find out available interaction options for the ligand at the receptor binding sites.

\section{Results \& Discussion}

\section{A. Bayesian modeling}

The cross-validated ROC plot (AUROCcv $=0.850)$ and the parameters, like specificity (0.932), sensitivity (0.710), concordance (0.840), and enrichment data (Table 1) of the developed Bayesian model (Model 1) indicate its ability to separate active and inactive compounds effectively. The five-fold cross-validation results AUCROCTest (0.759), validates the developed Bayesian model with the test set, signifies the acceptability of the model (Fig. 1). Top five favourable and unfavourable molecular features for AchE inhibitory activity were identified based on a Bayesian score using FCFP_6, shown in Fig. 2.

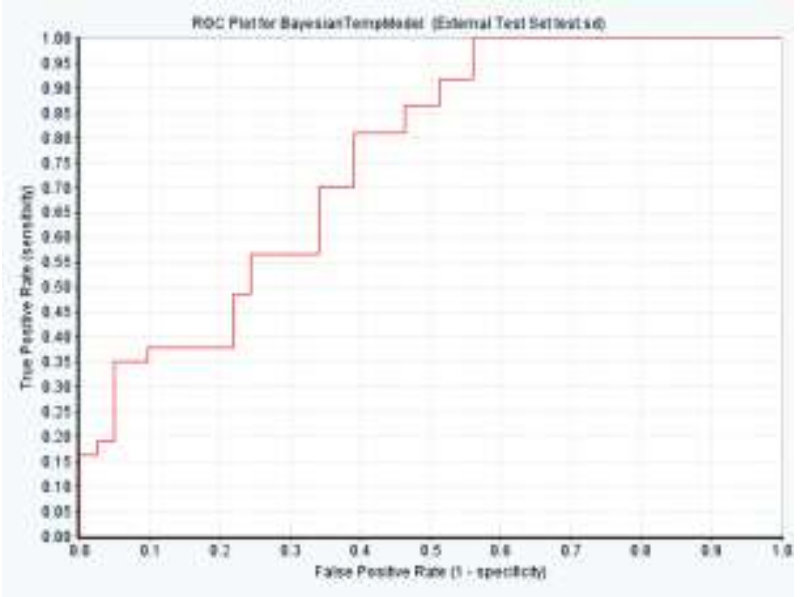

Figure 1. ROC plot for test set

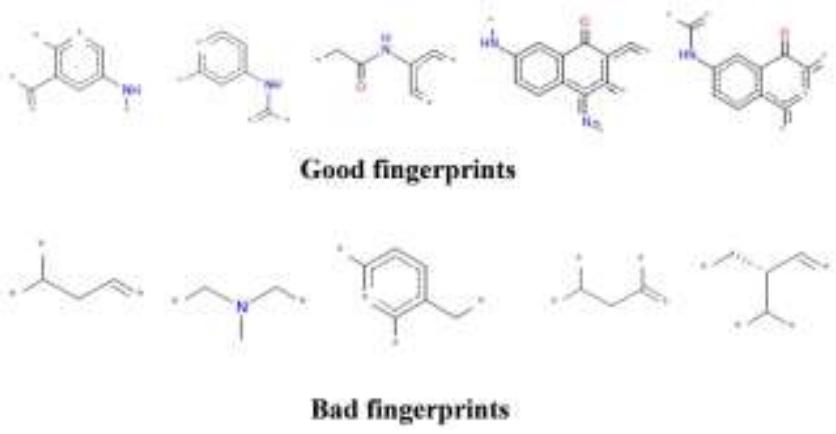

Figure 2. Good and bad fragments from the study

\section{B. Docking interaction}

The highly active compound was docked at the active site of the crystal structure, 4EY7 [18] (Fig. 3). The catalytic amino acids (within 4 Á) include Tyr72, Tyr341, Tyr337, (polar amino acids) and Phe295, Trp86, Trp286, His447, Gly448 (non-polar amino acids). Docking results satisfied the geometric criterion for HB formation and $\pi-\pi$ interaction. It is observed that HB- length is $\leq 2.8 \AA$, the minimum angle of donor $\geq 120^{\circ}$ and acceptor $\geq 90^{\circ}$, and piinteraction: bond length $4.4-5.5 \AA$ and angle $30^{\circ}-60^{\circ}$. The interaction study showed that carbonyl group of adjacent anthracen scaffold interacted with Phe295, and carbonyl group of the backbone chain interacted through the HB with Tyr124. The residues Trp286 and Tyr341 formed the $\pi-\pi$ stacking interactions and Trp 86 formed the $\pi$-cation interaction. But the least active compound didn't show any interaction with Phe 295 which is important for the activity.

\section{Site map analysis}

To explore structure-based QSAR study, Site Map is generated at the active site of the AChE enzyme through the docked pose of ligand. The ligand bound pocket of the protein structure showed good druggability (SiteScore: 1.006, Dscore: 1.050) score. Red color indicates HB acceptor region. Blue color indicates $\mathrm{HB}$ donor region and yellow color indicates hydrophobic region. Oxygen atom attached to five membered ring acts as a HB acceptor in red contour. Blue contour near electronegative atoms nitrogen and oxygen signifies the region HB donor. Alkane chains and benzene rings depict hydrophobic property for its nonpolarity (Fig. 4).

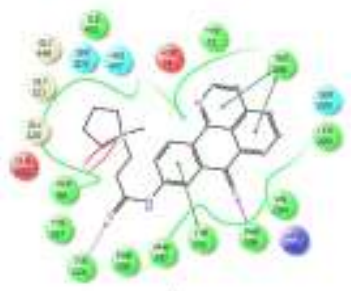

A
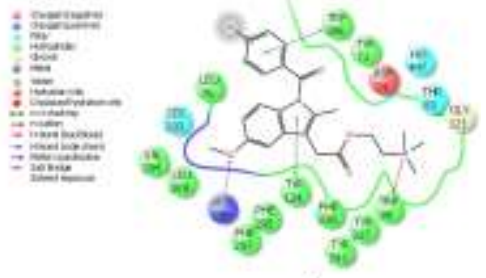

B
Figure 3. Docked poses of (A) most and (B) least active compounds. 
Proc. of the International Conference on Advances in Bio-Informatics and Environmental Engineering - ICABEE 2016. Copyright (c) Institute of Research Engineers and Doctors. All rights reserved.

ISBN: 978-1-63248-100-9 doi: 10.15224/ 978-1-63248-100-9-17

Table 1. Enrichment data

\begin{tabular}{|c|c|c|c|c|c|c|c|c|c|c|}
\hline Output & Category \% & $\mathbf{1 \%}$ & $\mathbf{5 \%}$ & $\mathbf{1 0 \%}$ & $\mathbf{2 5 \%}$ & $\mathbf{5 0 \%}$ & $\mathbf{7 5 \%}$ & $\mathbf{9 0 \%}$ & $\mathbf{9 5 \%}$ & $\mathbf{9 9 \%}$ \\
\hline $\begin{array}{c}\text { Bayesian } \\
\text { Model }\end{array}$ & $41.33 \%$ & $3.20 \%$ & $12.90 \%$ & $25.80 \%$ & $53.20 \%$ & $77.40 \%$ & $91.90 \%$ & $100 \%$ & $100 \%$ & $100 \%$ \\
\hline
\end{tabular}

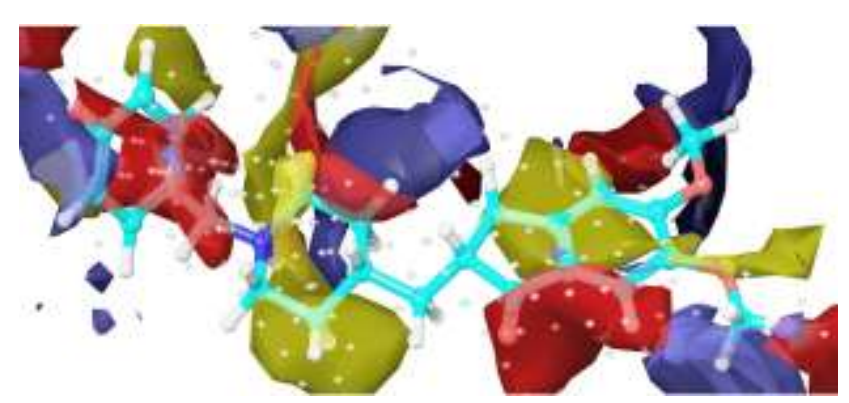

Figure 4. Site map features in AChE catalytic site. Blue: Donor, Red: acceptor and Yellow: hydrophobic features.

\section{Conclusion}

In the present study, both ligand-based and structurebased QSAR studies have performed on a structurally diverse AChE inhibitors and receptor cavity. Ligand-based QSAR study provides information on some essential groups as well as fragments, and structure-based QSAR study reveals vital feature sites in the active site cavity, which are important for binding inhibition to the receptor. Systematic comparisons revealed that structure-based QSAR has advantages in efficiently identifying potent hits with structural diversity over simple ligand-based QSAR. Both types of studies confirm the importance of HB acceptor, and donor with hydrophobic features. Presence of electronegative groups, and acyclic and aromatic rings in the molecular scaffold depicts their importance in AChE inhibition.

\section{Authors and Affiliations}

Department of Chemical Technology, University of Calcutta, 92, A.P.C. Road, Kolkata-700009, India

\section{Acknowledgment}

Tabassum Hossain wishes to thank UGC-MANF for awarding Senior Research Fellowship and TEQIP Phase-II, University of Calcutta for financial assistance.

\section{References}

[1] A. Sadiq, F. Mahmood, F. Ullah, M. Ayaz, S. Ahmad, F. Haq, G. Khan, and M. Jan, "Synthesis, anticholinesterase and antioxidant potentials of ketoesters derivatives of succinimides: a possible role in the management of Alzheimer's," Chem. Cent. J., vol. 9, pp. 31, May 2015 .

[2] D. Shao, C. Zou, C. Luo, X. Tang, and Y. Li, "Synthesis and evaluation of tacrine-E2020 hybrids as acetylcholinesterase inhibitors for the treatment of Alzheimer's disease," Bio. Med. Chem. Lett., Vol. 14, pp. 4639-4642, July 2004.

[3] D. A. Bianchi, G. S. Hirschmann, C. Theoduloz, A. B. J. Bracca, and T. S. Kaufman, "Synthesis of tricyclic analogs of stephaoxocanidine and their evaluation as acetylcholinesterase inhibitors," Bio. Med. Chem. Lett., vol. 15, pp. 2711-2715, April 2005.
[4] S. Young, K. Fabio, C. Guillon, P. Mohanta, T. A. Halton, D. E. Heck, R. A. Flowers II, J. D. Laskin, and N. D. Heindel, "Peripheral site acetylcholinesterase inhibitors targeting both inflammation and cholinergic dysfunction," Bio. Med. Chem. Lett., vol. 20, pp. 29872990, February 2010.

[5] L. Pan, J. H. Tan, J. Q. Hou, S. L. Huang, L. Q. Gu, and Z. S. Huang, "Design, synthesis and evaluation of isaindigotone derivatives as acetylcholinesterase and butyrylcholinesterase inhibitors," Bio. Med. Chem. Lett., vol. 18, pp. 3790-3793, May 2008.

[6] M. Schulze, O. Siol, M. Decker, and J. Lehmann, "Bivalent 5,8,9,13btetrahydro-6H-isoquino[1,2-a]isoquinolines and -isoquinolinium salts: Novel heterocyclic templates for butyrylcholinesterase inhibitors," Bio. Med. Chem. Lett., vol. 20, pp. 2946-2949, March 2010.

[7] H. Wen, Y. Zhou, C. Lin, H. Ge, L. Ma, Z. Wang, W. Peng, and H. Song, "Methyl 2-(2-(4-formylphenoxy)acetamido)-2-substituted acetate derivatives: A new class of acetylcholinesterase inhibitors," Bio. Med. Chem. Lett., vol. 17, pp. 2123-2125, January 2007.

[8] R. Palin, J.K. Clark, P. Cowley, A. W. Muir, E. Pow, A. B. Prosser, R. Taylor, and M. Q. Zhang, "Novel Piperidinium and Pyridinium Agents as Water-Soluble Acetylcholinesterase Inhibitors for the Reversal of Neuromuscular Blockade," Bio. Med. Chem. Lett., vol. 12, pp. 2569-2572, June 2002.

[9] L. Huang, Z. Luo, F. He, A. Shi, F. Qin, and X. Li, "Berberine derivatives, with substituted amino groups linked at the 9-position, as inhibitors of acetylcholinesterase/butyrylcholinesterase," Bio. Med. Chem. Lett., vol. 20, pp. 6649-6652, September 2010.

[10] H. Tang, F. X. Ning, Y. B. Wei, S. L. Huang, Z. S. Huang, A.S.C. Chan, and L. Q. Gu, "Derivatives of oxoisoaporphine alkaloids: A novel class of selective acetylcholinesterase inhibitors," Bio. Med. Chem. Lett., vol. 17, pp. 3765-3768, April 2007.

[11] M. A. Ali, M. S. Yar, M. Z. Hasan, M. J. Ahsan, and S. Pandian, "Design, synthesis and evaluation of novel 5,6-dimethoxy-1-oxo-2,3dihydro-1H-2-indenyl-3,4-substituted phenyl methanone analogues," Bio. Med. Chem. Lett., vol. 19, pp. 5075-5077, July 2009.

[12] M. A. Ali, R. Ismail, T. S. Choon, Y. K. Yoon, A. C. Wei, S. Pandian, R. S. Kumar, H. Osman, and E. Manogaran, "Substituted spiro [2.3'] oxindolespiro [3.2"]-5,6-dimethoxy-indane-1"-one-pyrrolidine analogue as inhibitors of acetylcholinesterase," Bio. Med. Chem. Lett., vol. 20, pp. 7064-7066, September 2010.

[13] S. Y. Han, S. C. Mayer, E. J. Schweiger, B. M. Davis, and M. M. Joullie, "Synthesis and biological activity of galanthamine derivatives as acetylcholinesterase (AChE) inhibitors," Bio. Med. Chem. Lett., vol.1, pp. 579-580, August1991.

[14] J. Takahashi , I. Hijikuro, T. Kihara, M. G. Murugesh, S. Fuse, Y. Tsumura, A. Akaike, T. Niidome, T. Takahashi, and H. Sugimoto, "Design, synthesis and evaluation of carbamate-modified (-)-N1phenethylnorphysostigmine derivatives as selective butyrylcholinesterase inhibitors," Bio. Med. Chem. Lett., vol. 20, pp. 1721-1723, January 2010

[15] L. Yu, R. Cao, W. Yi, Q. Yan, Z. Chen, L. Ma, W. Peng, and H. Song, "Synthesis of 4-[(diethylamino)methyl]-phenol derivatives as novel cholinesterase inhibitors with selectivity towards butyrylcholinesterase," Bio. Med. Chem. Lett., vol. 20, pp. 32543258, April 2010

[16] C. T. Sadashiva, J. N. N. S. Chandra, K. C. Ponnappa, T. V. Gowda, and K. S. Rangappa, "Synthesis and efficacy of 1-[bis(4fluorophenyl)-methyl]piperazine derivatives for acetylcholinesterase inhibition, as a stimulant of central cholinergic neurotransmission in Alzheimer's disease” Bio. Med. Chem. Lett, vol. 16, pp. 3932-3936, May 2006.

[17] Y. Schott, M. Decker, H. Rommelspacher, and J. Lehmann, "6Hydroxy- and 6-methoxy-b-carbolines as acetyl- and butyrylcholinesterase inhibitors," Bio. Med. Chem. Lett, vol. 16, pp. 5840-5843, August 2006.,

[18] J. Cheung, M. J. Rudolph, F. Burshteyn, M. S. Cassidy, E. N. Gary, J. Love, M. C. Franklin, and J. J. Height, "Structures of human 
Proc. of the International Conference on Advances in Bio-Informatics and Environmental Engineering - ICABEE 2016.

Copyright (C) Institute of Research Engineers and Doctors. All rights reserved.

ISBN: 978-1-63248-100-9 doi: 10.15224/ 978-1-63248-100-9-17

acetylcholinesterase in complex with pharmacologically important ligands," J. Med. Chem., vol. 55, pp. 10282-10286, October 2012.

[19] RCSB Protein Data Bank. http://www.rcsb.org/pdb/.

[20] A. E. Klon, J. F. Lowrie, and D. J. Diller, "Improved naïve Bayesian modeling of numerical data for absorption, distribution, metabolism and excretion (ADME) property prediction," J. Chem. Inf. Model, vol. 46, pp. 1945-1956, July 2006.

[21] P. Prathipati, N. L. Ma, and T. H. Keller, "Global Bayesian models for the prioritization of antitubercular agents," J. Chem. Inf. Model, vol. 48, pp. 2362-2370, November 2008.

[22] DISCOVERY STUDIO 2.5. 2009; Accelrys Inc., SanDiego.

[23] R. A. Friesner, J. L. Banks, R. B. Murphy, T. A. Halgren, J. J. Klicic, D. T. Maint, and P. S. Shenkin, "Glide:A new approach for rapid, accurate docking and scoring. 1. Method and assessment of docking accuracy," J. Med. Chem., Vol. 47, pp. 1739-1749, February 2004.

[24] T. A. Halgren, R. B. Murphy, R. A. Friesner, H. S. Beard, L. L. Frye, W. T. Pollard, and J. L. Banks, "Glide: A new approach for rapid, accurate docking and scoring. 2. Enrichment factors in database screening,". J. Med. Chem., vol. 47, pp. 1750-1759, February 2004.

[25] C. Selvaraj, and S.K. Singh, "Validation of potential inhibitors for SrtA against Bacillus anthracis by combined approach of ligandbased and molecular dynamics simulation," J. Biomol. Struct. Dyn., vol. 32, pp. 1333-1349, July 2014.

[26] SiteMap 2.9. 2013; Schrödinger, LLC, New York, NY.

[27] P. J. Goodford, "A computational procedure for determining energetically favourable binding sites on biologically important macromolecules," J. Med. Chem., vol. 28, pp. 849-857, July 1985.
About Author (s):

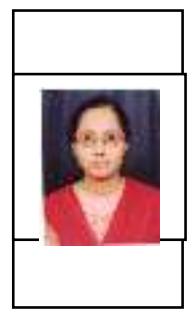

Tabassum Hossain, M. Sc. in Bioinformatics, is pursuing Ph.D in the University of Calcutta as a Senior Research Fellow under the Moulana Azad National Fellowship of University Grant Commission, India. She has published 8 research papers and presented a research article in 5th FIP Pharmaceutical Sciences World Congress 2014 at Melbourne, Australia.

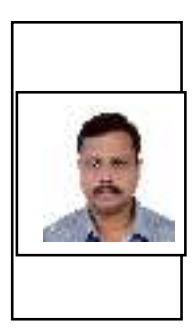

Dr. Achintya Saha, Professor and Head of the Department of Chemical Technology, University of Calcutta, has supervised ten $\mathrm{Ph} . \mathrm{D}$ students and guided eight research projects funded by different organizations. He has published more than 100 papers in standard journals. He was awarded SAARC fellowship in 2013. 